\title{
Joint Space Redundancy Resolution of Serial Link Manipulator: An Inverse Kinematics and Continuum Structure Numerical Approach
}

\author{
Toshit Jain ${ }^{1}$, Jinesh Kumar Jain ${ }^{1}$, Debanik Roy ${ }^{2}$ \\ ${ }^{I}$ Department of Mechanical Engineering, Malaviya National Institute of Technology, Jaipur, India \\ ${ }^{2}$ Homi Bhabha National Institute (HBNI), Department of Atomic Energy (DAE), Mumbai 400085, India \\ 2019rme9036@mnit.ac.in, jineshjain.mech@mnit.ac.in
}

Dr Jinesh Kumar Jain is currently working as Associate Professor at Malaviya National Institute of Technology Jaipur (MNIT) having research interests in Joining and Welding, Additive Manufacturing and Biofabrication, Design and Manufacturing Technology, Operation Management, Sustainable Manufacturing. He completed his PhD and M.Tech. from MNIT and B.E. from MBM Engineering College Jodhpur. He has more than twenty years of teaching, research and industrial experience and published many technical papers in journals and conference proceedings and delivered lectures in reputed institutions in India and abroad. Prior to joining MNIT he has worked in several organizations. Dr Jain is a life member of many reputed professional bodies and board member in number of Indian universities. Dr Jain successfully guided many M.Tech dissertations and presently guiding many doctoral and masters scholars. He successfully organized many short term courses and faculty development programmes. Dr Jain authored number of books.

Mr Toshit Jain is currently pursuing his doctoral from MNIT and working as Assistant Professor at GLA University Mathura. He has research interests in Biofabrication, Biomaterials, Additive Manufacturing, Manufacturing and Automation, Robotics and AI. He completed his M.Tech from Nirma University, Ahmedabad and B.Tech. from GGSIPU Delhi. He has 5 years of teaching and research experience and published several technical papers in journals and conference proceedings and is a member of several professional bodies. He successfully guided many B. Tech and M.Tech dissertations. 


\title{
Joint Space Redundancy Resolution of Serial Link Manipulator: An Inverse Kinematics and Continuum Structure Numerical Approach
}

\begin{abstract}
Automatic control to any of robot manipulators, some kind of issues are being observed. A numerical method for solution generation to the inverse kinematics problem of redundant robotic manipulators is presented to obtain the smoothest algorithm as possible, leading to a robust iterative method. After the primary objective of the reachability of end-effectors to the target point is achieved, the aim is set to resolve the redundant degrees of freedom of redundant manipulator. This method is numerically stable since it converges to the correct answer with virtually any initial approximation, and it is not sensitive to the singular configurations of the manipulator. In addition, this technique is computationally effective and able to apply for serial manipulators with any DOF applications. A planar 3R-DOF serial link redundant manipulator is considered as exemplar problem for solving. Also, the continuum approach for resolving more complex structure with variable DoF is illustrated here and their brief applicability to support surgeries and adaptive use of artificial linkage moments is also calculated.
\end{abstract}

Keywords- inverse kinematics; DLS system; redundancy resolution; redundant manipulator; continuum structure; manipulator positioning

\section{Introduction}

Redundant manipulators have more kinematic DOFs than those are required to span a task space, producing an under-constrained problem. In the case of two-dimensional planar manipulation with both position and orientation targets, this means that a manipulator has four or more degrees of freedom, allowing a large number of manipulator joint-space solutions for a given task space position and orientation. The redundant manipulators have to face lack of solution uniqueness.

In the last 250 years, continuum mechanics filaments are been reinvented numerous times in recent past. Euler examined these objectives in eighteenth-century and later in nineteenth-century Kirchhoff and Clebsch gauged these objectives, and in early twenty century, F. and E. Cosserat presented his nonlinear theory regarding rods.

\section{II.Literature work}

Since the beginning of robotics, since the evolution of robotics and built of robot Inverse kinematics algorithms are a major concern for a generation of differential paths. To overcome this issue, analytical methods were the most popular methods. Often, a unique solution does not always exist, and then it becomes necessary to find an alternate solution. The most extensively used methods are the numerical ones, however, there are several methods related to learning, neural networks are available [1], interval methods [2], or distance associated [3]. On control-based methods, the first approaches were developed in the 60s-70s, when

Whitney [4] used the pseudo-inverse of a matrix for solving the problem. However other methods like Jacobian transpose, relatively faster, were also used. Earlier in the 1980s, with the developing computational capabilities of computers, Baillieul[5] presented an extended Jacobian, 
considered more distinctive computationally, with second-order derivatives which was further developed in 1995[6]. Also in the 80s, the manipulability index was introduced by Yoshikawa [7] and optimizing parameter for a redundant robot. Some techniques of avoiding discontinuities on the singularities by damping/ filtering the Jacobian Matrix are discussed in [8,9]. Many ways of improving the performance of these algorithms have been proposed. Siciliano and Slotine presented in 1991 away to prioritize various tasks [9, 10] and analyzed the damping of the Jacobian to avoid the discontinuity of the pseudo-inverse operator for the rank deficient Jacobian matrix with its alternatives[11].

Hirose and substitutes arose the concept study of snake structured robots at the beginning of the 1970s[12, 13]. Then after such snakelike locomotive systems[14] are designed and developed for search-and-rescue types of operations[15, 16]. Elastic-filament type of manipulators are mainly used in orienting and positioning of objects, also used for carrying nasal and throat type surgeries $[17,18]$. Continuum[19, 20] and concentric elastic types of tube robotics [21] have introduced for variability in jobs comprising medical techniques. Hyper-redundant manipulators are also introduced in satellite servicing and inspection to be used in nuclear power plants.

Continuum manipulators gained popularity in nearby years[15][17] including grasping applications [22, 23]. Often, researchers in this field point to as the starting point for the study of such manipulators.

The continuous backbone curves are applicable either for hyper-redundant manipulators having discrete or continuous (aka continuum) morphologies. For continuing the research interest problem bio-inspired type structures like tentacles $[20,21]$ and Locomotion operations $[3,25,26]$ can be used.

Using a medical point of interpretation these teleoperated surgical robotics able to provide a better and precise motion simulation of instrumentation for variable controlling capabilities [27] [28]. Whereas in general robotics working principally requires operating space in the large-spectrum, needs to be rigid and positioned tip. Hence, tending towards evident invasive surgery, it requires more trending areas which are challenging, delicate and circuitous to be done in an open approach $[29,30]$. For such results development of manipulators in the smaller size, access difficult terrain and moving in crucial pathways in surgery operations with dexterity [31,32]. A brief introduction of such robot applications and motion complexities illustrated here. The continuum robot's multibackbone structure consists of relatively constrained and parallel elastic elements (mainly tubes or rods) in an explicit manner.

Simaan et. Al.[15, 16] accumulated the designs theories consisting of central and secondary backbone/bending sec. proving taper using spacer and corner fixed to disks of sections[33].

The Concentric-tube type of robots is developed using internally nested pre-curved elastic tubes where base to each can be liable to rotate and transverse for proving the differential structure of robots. They are mainly made up of NiTi alloy (also acts as shape memory polymer) at super elastic constituent phase through which desired forms can be gained by providing heat constituents before the concentric assembly of tubes [34-36]. 
For structurally dynamic mechanism stiffness and robot range and design, motion is considered. Here workspace and linked stiffness must be kept large to make the movement for conquering destination and adequate forces can be imparted to accumulate the required results on the provided payload [37].

Although its actuation is termed under intrinsic and extrinsic, in lieu of type of motion and forces applied, for actuation to provide motion within structure intrinsic and to provide structure actuation with forces tor transmitting mechanical bodies' extrinsic type is used [38]. This can be provided using electromagnetic motors by translating base of cable to the outside of manipulator (extrinsic) and shortening or shrinking of each cable length providing shape memory properties (intrinsic type) [39].

Energy methods are also important criteria applicable in the number of purposes under the continuum approach [40,41]. Minimizing elastic potential is an important constituent in hyper redundancy (Euler Lagrange energy equations) for the continuous framework.

Invasive surgery mainly relies on surface interaction anatomy and imagining [42, 43]. A transition has done between firm endoscopes providing high visualization and flexibility using bending tips at lower visualization [44]. Da-Vinci surgical which are highly definitive is prioritize by surgeons for important depth mechanics consideration [45]. For delivering instrumental safety to operative patient manipulator compliance is a very important aspect in designing and considerations [46, 47]. But in case of contact forces for tissues contact forces and moments for robot deflection for drawing anatomical aspects in motion planning for elimination of uneven and non-desirable deflections[3, 25].

Later by viably validate the desired parameters and later simulation, the addition of man-made or nature-inspired materials can be introduced in the fabrication of such parts [37, 48] Although this approach shows much resemblance in squid tentacle simulation and their motion studies where connecting tissue parameters can be replaced with rubber and EAP type artificial muscles[49].

\section{Methodology of Inverse Kinematics}

\section{The transformation between Two Adjacent Frames}

In this convention, each homogeneous transformation ${ }^{i-1} T_{i}$ (homogeneous transformation of frame i [1-58] with respect to frame $\{i-1\})$ is represented as a product of four basic transformations:

$$
\begin{array}{r}
{ }^{i-1} T_{i}\left(\mathrm{q}_{\mathrm{i}}\right)=\operatorname{Rot}\left(\theta_{\mathrm{i}}, \mathrm{Z}_{\mathrm{i}-1}\right) \cdot \operatorname{Trans}\left(\mathrm{d}_{\mathrm{i}}, \mathrm{Z}_{\mathrm{i}-1}\right) \cdot \operatorname{Trans}\left(\mathrm{a}_{\mathrm{i}}, \mathrm{X}_{\mathrm{i}}\right) \cdot \operatorname{Rot}\left(\alpha_{\mathrm{i}}, \mathrm{X}_{\mathrm{i}}\right)(2.1) \\
=\left[\begin{array}{cccc}
\cos \theta_{i} & \sin \theta_{i} \cos \alpha_{i} & -\sin \theta_{i} \sin \alpha_{i} & a_{i} \cos \theta_{i} \\
\sin \theta_{i} & \cos \theta_{i} \cos \alpha_{i} & -\cos \theta_{i} \sin \alpha_{i} & a_{i} \sin \theta_{i} \\
0 & \sin \alpha_{i} & \cos \alpha_{i} & d_{i} \\
0 & 0 & 0 & 1
\end{array}\right]
\end{array}
$$

\subsection{Numerical Solution of the Inverse Kinematic Problem}


The project work is completely based on numerical computation methods in MATLAB. In most practical situations manipulators are redundant. In such cases, the closed-form solution is not possible. Therefore, the methods are iterative. The most common method Newton-Raphson method is adopted here.

In the iterative technique, we solve the kinematic equations

$$
\begin{aligned}
& x=f(q) \\
& f(q)-x=0 \\
& F(q)=0 \quad(\text { assume } f(q)-x=F(x))
\end{aligned}
$$

For variables $\mathrm{q}$, we start with an initial guess $\quad \mathrm{q}^{*}=\mathrm{q}+\delta \mathrm{q}$

For the guessed joint variables, we can determine the configuration of the end-effector frame using the forward kinematics.

$$
F^{*}=F\left(q^{*}\right)
$$

The difference between the calculated configuration using forward kinematics and the target configuration.

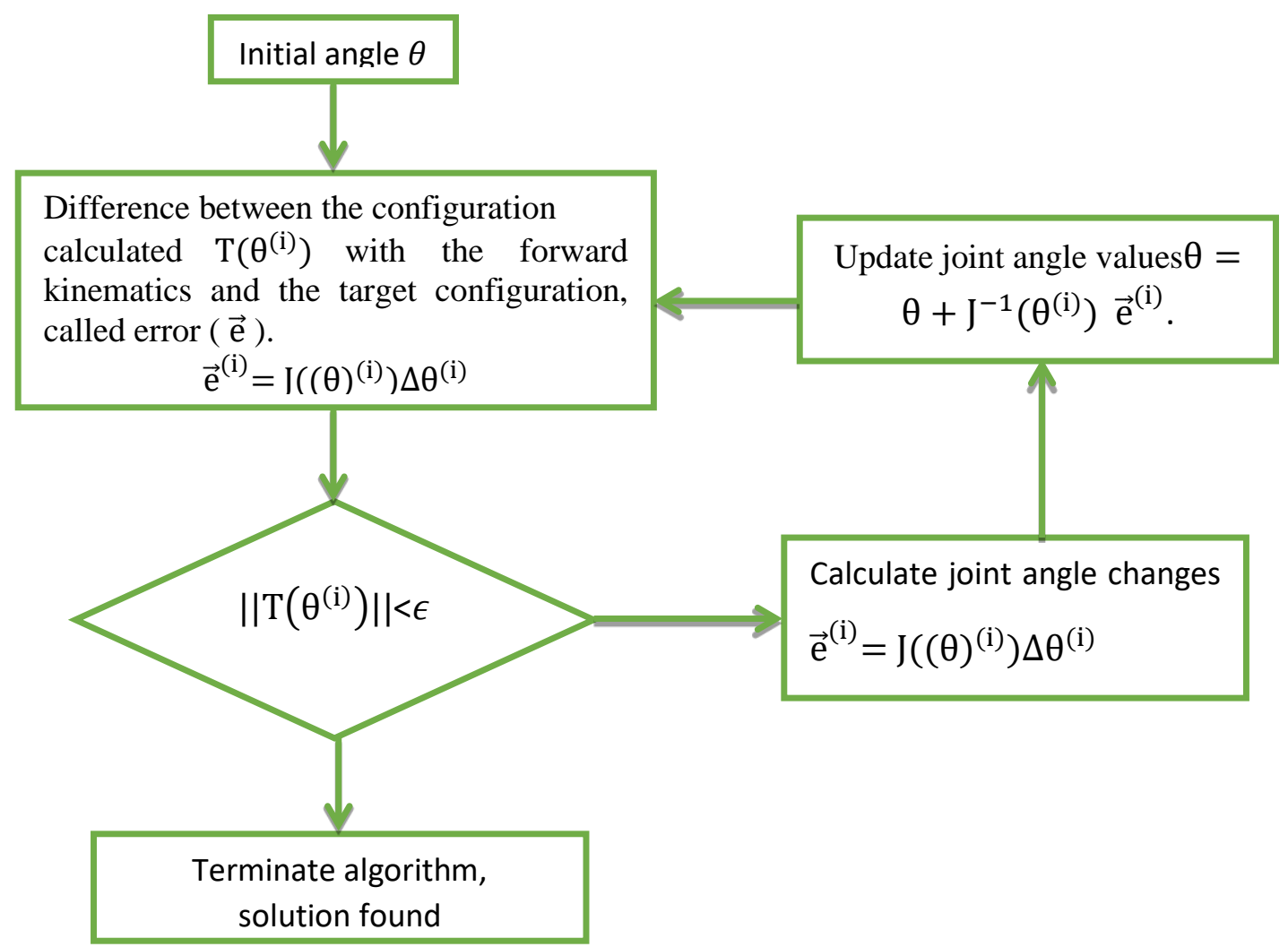

Fig. 1: Diagram of the Newton-Raphson Algorithm used for IK Calculations

\subsubsection{Pseudo-inverse method}

In the above algorithm in step 5 while updating joint angle values inverse of the jacobian matrix is required to be calculated. The inverse can be defined only for an invertible matrix i.e. the square and nonsingular matrix. The extension of inverse operation for non-singular matrices is known as the Moore-Penrose pseudo-inverse or pseudo-inverse. If $\mathrm{A}$ is invertible, then the Moore-Penrose pseudo-inverse is equal to the matrix inverse.

Mathematical formula:

From forward kinematics of robot manipulator, we have 


$$
\mathrm{J} \Delta \theta=\overrightarrow{\mathrm{e}}
$$

The pseudo-inverse method can be derived as follows:

$$
\mathrm{J}^{\mathrm{T}} \mathrm{J} \Delta \theta=\mathrm{J}^{\mathrm{T}} \overrightarrow{\mathrm{e}}
$$

After solving inverse kinematics to find required joint angles, we can write

$$
\begin{gathered}
\Delta \theta=\mathrm{J}^{+} \overrightarrow{\mathrm{e}} \\
\mathrm{J}^{+}=\mathrm{J}^{\mathrm{T}}\left(\mathrm{J} \mathrm{J}^{\mathrm{T}}\right)^{-1}
\end{gathered}
$$

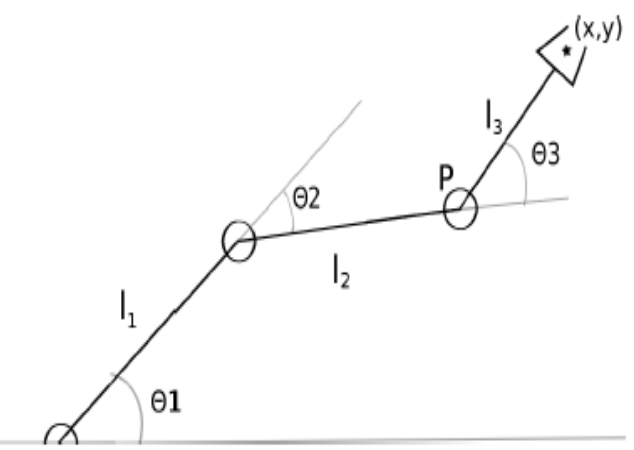

Fig. 2: 3R Planar Manipulator [14]

Table 1 : DH Parameter Table for 3R Planar Manipulator

\begin{tabular}{|c|c|c|c|c|}
\hline \multirow{2}{*}{$\begin{array}{l}\text { Link No. / Joint No } \\
\text { ( i })\end{array}$} & \multicolumn{2}{|c|}{ Joint Parameter } & \multicolumn{2}{|c|}{ Link Parameter } \\
\cline { 2 - 5 } & $\theta_{\mathrm{i}}$ & $\mathrm{d}_{\mathrm{i}}$ & $\mathrm{a}_{\mathrm{i}}$ & $\alpha_{\mathrm{i}}$ \\
\hline 1 & $\theta_{1}$ & 0 & $\mathrm{l}_{1}$ & 0 \\
\hline 2 & $\theta_{2}$ & 0 & $\mathrm{l}_{2}$ & 0 \\
\hline 3 & $\theta_{3}$ & 0 & $\mathrm{l}_{3}$ & 0 \\
\hline
\end{tabular}

$$
\begin{aligned}
{ }^{0} \mathrm{~T}_{1} & =\left[\begin{array}{cccc}
\cos \theta_{1} & -\sin \theta_{1} & 0 & l_{1} \cos \theta_{1} \\
\sin \theta_{1} & \cos \theta_{1} & 0 & l_{1} \sin \theta_{1} \\
0 & 0 & 1 & 0 \\
0 & 0 & 0 & 1
\end{array}\right] \\
{ }^{1} \mathrm{~T}_{2} & =\left[\begin{array}{cccc}
\cos \theta_{2} & -\sin \theta_{2} & 0 & l_{2} \cos \theta_{2} \\
\sin \theta_{2} & \cos \theta_{2} & 0 & l_{2} \sin \theta_{2} \\
0 & 0 & 1 & 0 \\
0 & 0 & 0 & 1
\end{array}\right] \\
{ }^{2} \mathrm{~T}_{3} & =\left[\begin{array}{cccc}
\cos \theta_{3} & -\sin \theta_{3} & 0 & l_{3} \cos \theta_{3} \\
\sin \theta_{3} & \cos \theta_{3} & 0 & l_{3} \sin \theta_{3} \\
0 & 0 & 1 & 0 \\
0 & 0 & 0 & 1
\end{array}\right]
\end{aligned}
$$




$$
{ }^{0} \mathrm{~T}_{3}={ }^{0} \mathrm{~T}_{1} \cdot{ }^{1} \mathrm{~T}_{2} \cdot{ }^{2} \mathrm{~T}_{3}
$$

The equation gives a transformation matrix of end-effector's frame w.r.t base frame. Hence last column vector of this transformation matrix gives coordinates of end-effector in Cartesian space.

Co-ordinates of end-effector

$\mathrm{x}=\mathrm{l}_{1} \cos \left(\theta_{1}\right)+\mathrm{l}_{2} \cos \left(\theta_{1}+\theta_{2}\right)+\mathrm{l}_{3} \cos \left(\theta_{1}+\theta_{2}+\theta_{3}\right)$

$$
\mathrm{y}=\mathrm{l}_{1} \sin \left(\theta_{1}\right)+\mathrm{l}_{2} \sin \left(\theta_{1}+\theta_{2}\right)+\mathrm{l}_{3} \sin \left(\theta_{1}+\theta_{2}+\theta_{3}\right)
$$

$=\left[\begin{array}{lll}\frac{\partial \mathrm{T}_{1}}{\partial \theta_{1}} & \frac{\partial \mathrm{T}_{1}}{\partial \theta_{2}} & \frac{\partial \mathrm{T}_{1}}{\partial \theta_{3}} \\ \frac{\partial \mathrm{T}_{2}}{\partial \theta_{1}} & \frac{\partial \mathrm{T}_{2}}{\partial \theta_{2}} & \frac{\partial \mathrm{T}_{2}}{\partial \theta_{3}}\end{array}\right]$

$$
\begin{gathered}
\mathrm{T}(\theta)=\left[\begin{array}{l}
\mathrm{X} \\
\mathrm{y}
\end{array}\right]=\left[\begin{array}{l}
\mathrm{T}_{1} \\
\mathrm{~T}_{2}
\end{array}\right] \\
J=\frac{\partial \mathrm{T}}{\partial \theta}
\end{gathered}
$$

$\frac{\partial \mathrm{T}_{1}}{\partial \theta_{1}}=-\mathrm{l}_{1} \sin \left(\theta_{1}\right)-\mathrm{l}_{2} \sin \left(\theta_{1}+\theta_{2}\right)-\mathrm{l}_{3} \sin \left(\theta_{1}+\theta_{2}+\theta_{3}\right)$

$\frac{\partial \mathrm{T}_{1}}{\partial \theta_{2}}=-\mathrm{l}_{2} \sin \left(\theta_{1}+\theta_{2}\right)-\mathrm{l}_{3} \sin \left(\theta_{1}+\theta_{2}+\theta_{3}\right)$

$\frac{\partial \mathrm{T}_{1}}{\partial \theta_{3}}=-\mathrm{l}_{3} \sin \left(\theta_{1}+\theta_{2}+\theta_{3}\right)$

$\frac{\partial \mathrm{T}_{2}}{\partial \theta_{1}}=\mathrm{l}_{1} \cos \left(\theta_{1}\right)+\mathrm{l}_{2} \cos \left(\theta_{1}+\theta_{2}\right)+\mathrm{l}_{3} \cos \left(\theta_{1}+\theta_{2}+\theta_{3}\right)$

$\frac{\partial \mathrm{T}_{2}}{\partial \theta_{2}}=\mathrm{l}_{2} \cos \left(\theta_{1}+\theta_{2}\right)+\mathrm{l}_{3} \cos \left(\theta_{1}+\theta_{2}+\theta_{3}\right)$

$\frac{\partial \mathrm{T}_{2}}{\partial \theta_{3}}=\mathrm{l}_{3} \cos \left(\theta_{1}+\theta_{2}+\theta_{3}\right)$

Taking each link of $3 \mathrm{R}$ planar redundant manipulator equal to 2 units.

$$
\begin{aligned}
& \mathrm{l}_{1}=2 \text { units } \\
& \mathrm{l}_{2}=2 \text { units } \\
& \mathrm{l}_{3}=2 \text { units }
\end{aligned}
$$

The workspace of the manipulator is a circular plane of radius 6 units.

Taking a base frame of the manipulator as origin, inverse kinematics can be solved for each point in the workspace.

Consider a point $(2,4)$ within the workspace and if we solve for corresponding joint angles of manipulator links, the algorithm converges iteratively and finally, we get solution as:

First joint angle $\theta_{1}=88.41$

Second joint angle $\theta_{2}=-88.36$

Third joint angle $\theta_{3}=91.61$

All angles are in degree.

The pseudo-inverse method performs well inside the workspace.

It converges to the given target point $(2,4)$ iteratively as shown in the figure 3 : 


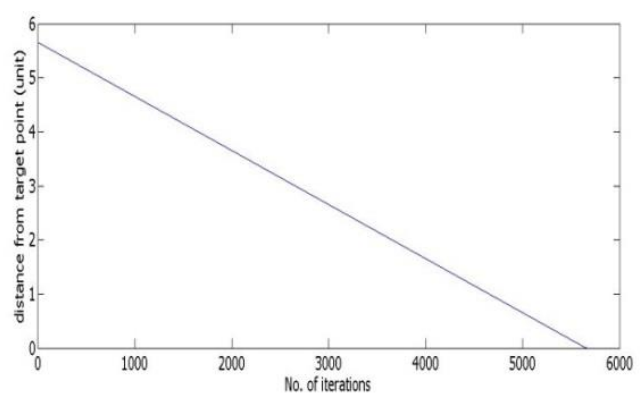

Fig. 3: Rate of Convergence of Inverse

\subsubsection{Kinematics Algorithm Using Pseudo-inverse method}

This method performs poorly near singular configurations due to instability as shown in figure no. 4:

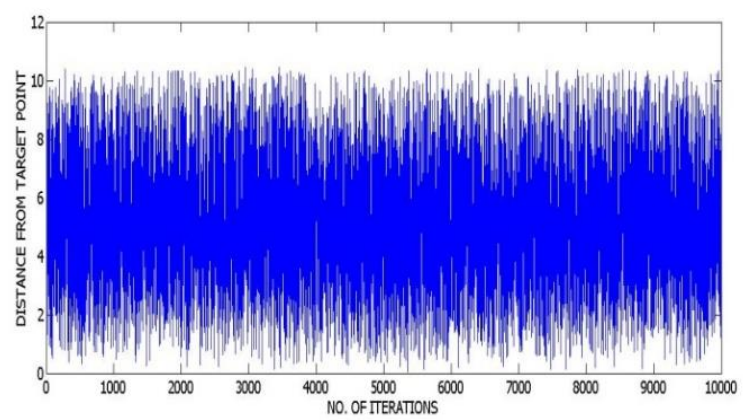

Fig. 4: Oscillating behaviour of Inverse Kinematics

Algorithm near singularities

Damped least-squares using Singular value decomposition

For the investigation of damped least square methods Singular Value Decomposition (SVD) comes out as a powerful technique.

A singular value decomposition of $\mathbf{J}$ comprises of stating $\mathbf{J}$ as representation

$$
\mathrm{J}=\mathrm{UDV}^{\mathrm{T}}
$$

Where $\mathrm{U}$ and $\mathrm{V}$ are orthogonal matrices and $D$ is a diagonal matrix with diagonal entries $\sigma_{\mathrm{i}}$. The singular value decomposition of $\mathbf{J}$ mainly subsists and entails that $J$ can be represented as

$$
\sum_{i=0}^{r} U D V ~ V^{T}
$$

Hence on simplification, we get the final damped least square solution in the form

$$
\Delta \theta=\left(\sum_{\mathrm{i}=0}^{\mathrm{r}} \frac{\sigma_{\mathrm{i}}}{\sigma_{\mathrm{i}}^{2}+\lambda^{2}} \mathrm{v}_{\mathrm{i}} \mathrm{u}_{\mathrm{i}}^{\mathrm{T}}\right) \overrightarrow{\mathrm{e}}
$$

At singular configuration $\sigma_{\mathrm{i}}=0$. But still due to the term $\frac{\sigma_{\mathrm{i}}}{\sigma_{\mathrm{i}}{ }^{2}+\lambda^{2}}, \Delta \theta$ is defined and is equal to zero. Hence even at singular configurations Damped least square method gives stable results. 


\section{Example:}

Consider the previous example, since the point $(2,4)$ is in the interior of the workspace algorithm converges rapidly to reach the target point as shown in figure 5:

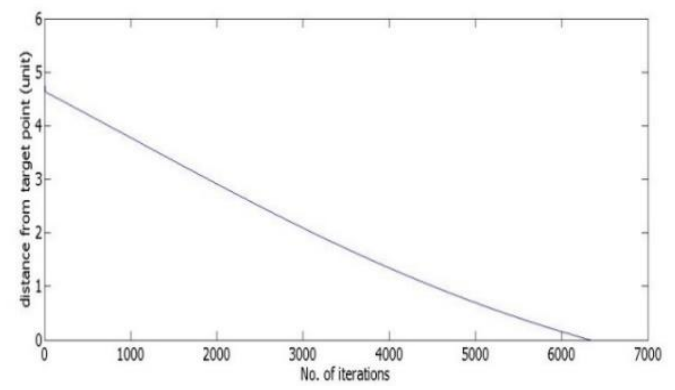

Fig. 5: Rate of Convergence of Inverse Kinematics

Algorithm Using the DLS method

\subsection{Effect of varying damping constant $(\lambda)$ on the algorithm:}

On increasing the value of $\lambda$, the convergence rate of the algorithm slows down. Hence computational time increases. This implies that no. of iterations also increases.

Now we will consider a particular point in the workspace and observe the behavior of the algorithm for different damping constants. Suppose the target point is to be reached at point $(2,4)$.

1. For $\lambda=0$,

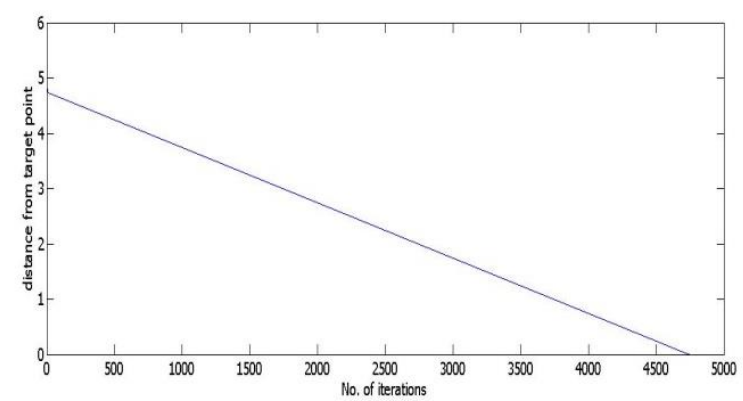

Fig. 6: Rate of Convergence of Inverse for $\lambda=0$

\subsubsection{Kinematics Algorithm Using the DLS method for $\lambda=0$}

When damping constant $\lambda=0$, the algorithm converges after 565 iterations as shown in figure 6 .

2. For $\lambda=1$,

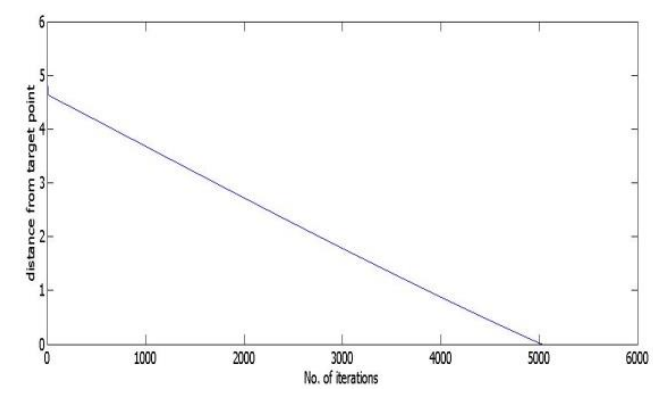

Fig. 7: Rate of Convergence of Inverse for $\lambda=1$ 


\subsubsection{Kinematics Algorithm Using the DLS method for $\lambda=1$}

When damping constant $\lambda=1$, the algorithm converges after 707 iterations (Figure 7).

\subsubsection{For $\lambda=5$,}

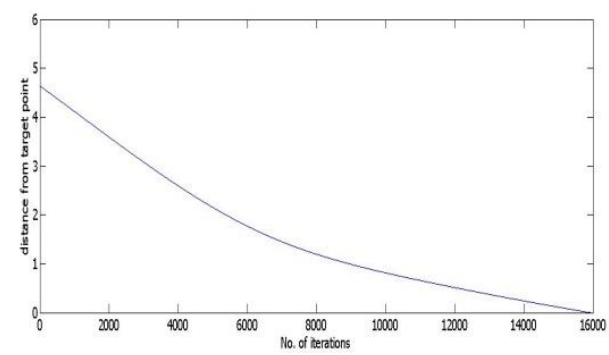

Fig. 8: Rate of Convergence of Inverse Kinematics

Algorithm Using DLS method for $\lambda=5$

When damping constant $\lambda=5$, the algorithm converges after 2779 iterations as shown in figure 8.

\subsubsection{For $\lambda=10$,}

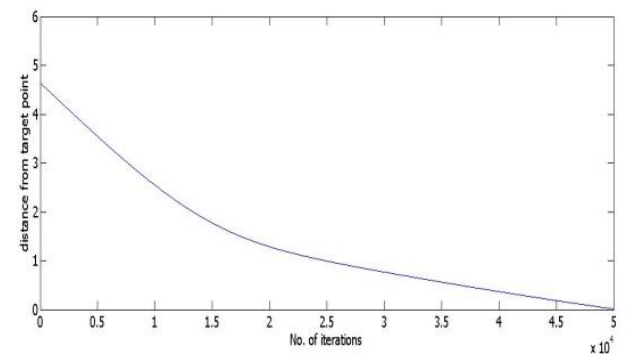

Fig. 9: Rate of Convergence of Inverse Kinematics

Algorithm Using the DLS method for $\lambda=10$

When damping constant $\lambda=10$, the algorithm converges after 9105 iterations as shown in figure 9.

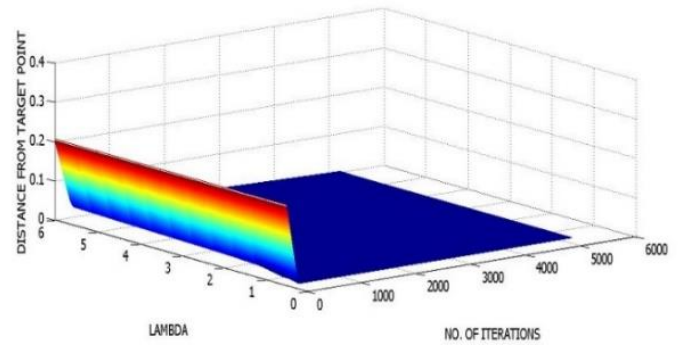

Fig. 10: Graph between damping constant $(\lambda)$, distance from target point (error ' $\mathrm{e}$ ') and no. of iterations in Damped Least Square Method 




Fig. 11: 3-D Plot of Convergence Rate with Varying Damping Constant

\section{Redundancy Resolution}

Redundancy resolution involves the use of manipulation systems which have more kinematic degrees of freedom than required to span a task space, producing an under-constrained problem. In the case of two-dimensional planar manipulation with both position and orientation targets, this means that a manipulator has four or more degrees of freedom.

\section{Manipulability Function}

Manipulability is the ability of a robotic mechanism in the positioning and is the orientation of the end-effector. Mathematically, manipulability (developed by Yoshikawa) can be expressed as [5]

$$
\text { Manipulability }(\mathrm{m})=\sqrt{\operatorname{det}\left(\mathrm{J} \cdot \mathrm{J}^{\mathrm{T}}\right)}
$$

\section{Maximization of Manipulability of 3R Planar Manipulator}

Considering a three degree of freedom planar redundant manipulator having three links with 2 units' length each.

Here joint variable (q) is joint angle $(\theta)$.

As we know the jacobian matrix

$$
\begin{gathered}
\mathrm{J}=\left[\begin{array}{lll}
\frac{\partial \mathrm{T}_{1}}{\partial \theta_{1}} & \frac{\partial \mathrm{T}_{1}}{\partial \theta_{2}} & \frac{\partial \mathrm{T}_{1}}{\partial \theta_{3}} \\
\frac{\partial \mathrm{T}_{2}}{\partial \theta_{1}} & \frac{\partial \mathrm{T}_{2}}{\partial \theta_{2}} & \frac{\partial \mathrm{T}_{2}}{\partial \theta_{3}}
\end{array}\right] \\
\mathrm{J}^{\mathrm{T}}=\left[\begin{array}{ll}
\frac{\partial \mathrm{T}_{1}}{\partial \theta_{1}} & \frac{\partial \mathrm{T}_{2}}{\partial \theta_{1}} \\
\frac{\partial \mathrm{T}_{1}}{\partial \theta_{2}} & \frac{\partial \mathrm{T}_{2}}{\partial \theta_{2}} \\
\frac{\partial \mathrm{T}_{1}}{\partial \theta_{3}} & \frac{\partial \mathrm{T}_{2}}{\partial \theta_{3}}
\end{array}\right]
\end{gathered}
$$

Objective function $\mathrm{w}(\theta)=\operatorname{det}\left(\mathrm{J} \mathrm{J}^{\mathrm{T}}\right)=\left|\begin{array}{ll}A 11 & A 12 \\ A 21 & A 22\end{array}\right|$ 


$$
\begin{aligned}
& =\left|\begin{array}{cc}
\left(\frac{\partial \mathrm{T}_{1}}{\partial \theta_{1}}\right)^{2}+\left(\frac{\partial \mathrm{T}_{1}}{\partial \theta_{2}}\right)^{2}+\left(\frac{\partial \mathrm{T}_{1}}{\partial \theta_{3}}\right)^{2} & \left(\frac{\partial \mathrm{T}_{1}}{\partial \theta_{1}}\right)\left(\frac{\partial \mathrm{T}_{2}}{\partial \theta_{1}}\right)+\left(\frac{\partial \mathrm{T}_{1}}{\partial \theta_{2}}\right)\left(\frac{\partial \mathrm{T}_{2}}{\partial \theta_{2}}\right)+\left(\frac{\partial \mathrm{T}_{1}}{\partial \theta_{3}}\right)\left(\frac{\partial \mathrm{T}_{2}}{\partial \theta_{3}}\right) \\
\left(\frac{\partial \mathrm{T}_{1}}{\partial \theta_{1}}\right)\left(\frac{\partial \mathrm{T}_{2}}{\partial \theta_{1}}\right)+\left(\frac{\partial \mathrm{T}_{1}}{\partial \theta_{2}}\right)\left(\frac{\partial \mathrm{T}_{2}}{\partial \theta_{2}}\right)+\left(\frac{\partial \mathrm{T}_{1}}{\partial \theta_{3}}\right)\left(\frac{\partial \mathrm{T}_{2}}{\partial \theta_{3}}\right) & \left(\frac{\partial \mathrm{T}_{2}}{\partial \theta_{1}}\right)^{2}+\left(\frac{\partial \mathrm{T}_{2}}{\partial \theta_{2}}\right)^{2}+\left(\frac{\partial \mathrm{T}_{2}}{\partial \theta_{3}}\right)^{2}
\end{array}\right| \\
& A 11=\left(\frac{\partial \mathrm{T}_{1}}{\partial \theta_{1}}\right)^{2}+\left(\frac{\partial \mathrm{T}_{1}}{\partial \theta_{2}}\right)^{2}+\left(\frac{\partial \mathrm{T}_{1}}{\partial \theta_{3}}\right)^{2} \\
& =\left(l_{1} \sin \left(\theta_{1}\right)+l_{2} \sin \left(\theta_{1}+\theta_{2}\right)+l_{3} \sin \left(\theta_{1}+\theta_{2}+\theta_{3}\right)\right)^{2}+\left(l_{2} \sin \left(\theta_{1}+\theta_{2}\right)+l_{3} \sin \left(\theta_{1}+\theta_{2}+\right.\right. \\
& \left.\left.\theta_{3}\right)\right)^{2}\left(l_{3} \sin \left(\theta_{1}+\theta_{2}+\theta_{3}\right)\right)^{2} \\
& \mathrm{~A}_{12}=\mathrm{A}_{21}=\left(\frac{\partial \mathrm{T}_{1}}{\partial \theta_{1}}\right)\left(\frac{\partial \mathrm{T}_{2}}{\partial \theta_{1}}\right)+\left(\frac{\partial \mathrm{T}_{1}}{\partial \theta_{2}}\right)\left(\frac{\partial \mathrm{T}_{2}}{\partial \theta_{2}}\right)+\left(\frac{\partial \mathrm{T}_{1}}{\partial \theta_{3}}\right)\left(\frac{\partial \mathrm{T}_{2}}{\partial \theta_{3}}\right) \\
& =\left[\left\{\left(\mathrm{l}_{1} \sin \left(\theta_{1}\right)+\mathrm{l}_{2} \sin \left(\theta_{1}+\theta_{2}\right)+\mathrm{l}_{3} \sin \left(\theta_{1}+\theta_{2}+\theta_{3}\right)\right) \times\left(\mathrm{l}_{1} \cos \left(\theta_{1}\right)+\mathrm{l}_{2} \cos \left(\theta_{1}+\right.\right.\right.\right. \\
& \left.\left.\left.\theta_{2}\right)+\mathrm{l}_{3} \cos \left(\theta_{1}+\theta_{2}+\theta_{3}\right)\right)\right\} \cdot\left\{( \mathrm { l } _ { 2 } \operatorname { s i n } ( \theta _ { 1 } + \theta _ { 2 } ) + \mathrm { l } _ { 3 } \operatorname { s i n } ( \theta _ { 1 } + \theta _ { 2 } + \theta _ { 3 } ) ) \cdot \left\{\left(\mathrm { l } _ { 2 } \operatorname { s i n } \left(\theta_{1}+\right.\right.\right.\right. \\
& \left.\left.\left.\theta_{2}\right)+\mathrm{l}_{3} \sin \left(\theta_{1}+\theta_{2}+\theta_{3}\right)\right) \times\left(\mathrm{l}_{2} \cos \left(\theta_{1}+\theta_{2}\right)+\mathrm{l}_{3} \cos \left(\theta_{1}+\theta_{2}+\theta_{3}\right)\right)\right\}\left(\mathrm { l } _ { 3 } \operatorname { s i n } \left(\theta_{1}+\theta_{2}+\right.\right. \\
& \left.\left.\left.\theta_{3}\right)\right) \times\left(l_{3} \cos \left(\theta_{1}+\theta_{2}+\theta_{3}\right)\right)\right]^{2} \\
& \mathrm{~A}_{21}=\left(\frac{\partial \mathrm{T}_{2}}{\partial \theta_{1}}\right)^{2}+\left(\frac{\partial \mathrm{T}_{2}}{\partial \theta_{2}}\right)^{2}+\left(\frac{\partial \mathrm{T}_{2}}{\partial \theta_{3}}\right)^{2} \\
& =\left(l_{1} \cos \left(\theta_{1}\right)+l_{2} \cos \left(\theta_{1}+\theta_{2}\right)+l_{3} \cos \left(\theta_{1}+\theta_{2}+\theta_{3}\right)\right)^{2}+\left(l_{2} \cos \left(\theta_{1}+\theta_{2}\right)\right) \cdot \cos \left(\theta_{1}+\right. \\
& \left.\left.\theta_{2}+\theta_{3}\right)\right)^{2}+\left(l_{3} \cos \left(\theta_{1}+\theta_{2}+\theta_{3}\right)\right)^{2} \\
& \mathrm{w}(\theta)=\left(\mathrm{l}_{1} \sin \left(\theta_{1}\right)+\mathrm{l}_{2} \sin \left(\theta_{1}+\theta_{2}\right)+\mathrm{l}_{3} \sin \left(\theta_{1}+\theta_{2}+\theta_{3}\right)+\left(\mathrm{l}_{2} \sin \left(\theta_{1}+\theta_{2}\right)+\mathrm{l}_{3} \sin \left(\theta_{1}+\right.\right.\right. \\
& \left.\left.\left.\theta_{2}+\theta_{3}\right)\right)^{2}+\left(l_{3} \sin \left(\theta_{1}+\theta_{2}+\theta_{3}\right)\right)^{2}\right)^{2} \times\left[\left(l_{1} \cos \left(\theta_{1}\right)+l_{2} \cos \left(\theta_{1}+\theta_{2}\right)+l_{3} \cos \left(\theta_{1}+\right.\right.\right. \\
& \left.\left.\left.\theta_{2}+\theta_{3}\right)\right)^{2}+\left(l_{2} \cos \left(\theta_{1}+\theta_{2}\right)+l_{3} \cos \left(\theta_{1}+\theta_{2}+\theta_{3}\right)\right)^{2}+\left(l_{3} \cos \left(\theta_{1}+\theta_{2}+\theta_{3}\right)\right)^{2}\right] \\
& -\left[\left\{\left(\mathrm{l}_{1} \sin \left(\theta_{1}\right)+\mathrm{l}_{2} \sin \left(\theta_{1}+\theta_{2}\right)+\mathrm{l}_{3} \sin \left(\theta_{1}+\theta_{2}+\theta_{3}\right)\right) \times\left(\mathrm{l}_{1} \cos \left(\theta_{1}\right)+\mathrm{l}_{2} \cos \left(\theta_{1}+\right.\right.\right.\right. \\
& \left.\left.\left.\theta_{2}\right)+\mathrm{l}_{3} \cos \left(\theta_{1}+\theta_{2}+\theta_{3}\right)\right)\right\}\left(\mathrm{l}_{2} \sin \left(\theta_{1}+\theta_{2}\right)+\mathrm{l}_{3} \sin \left(\theta_{1}+\theta_{2}+\theta_{3}\right)\right)+\left\{\left(\mathrm { l } _ { 2 } \operatorname { c o s } \left(\theta_{1}+\right.\right.\right. \\
& \left.\left.\left.\left.\theta_{2}\right)+\mathrm{l}_{3} \cos \left(\theta_{1}+\theta_{2}+\theta_{3}\right)\right)\right\} \cdot\left(\mathrm{l}_{3} \sin \left(\theta_{1}+\theta_{2}+\theta_{3}\right)\right) \times\left(\mathrm{l}_{3} \cos \left(\theta_{1}+\theta_{2}+\theta_{3}\right)\right)\right]^{2} \cdot\{1\} \\
& \mathrm{z}=\left\{\begin{array}{l}
\frac{\partial w(q)}{\partial \theta_{1}} \\
\frac{\partial w(q)}{\partial \theta_{2}} \\
\frac{\partial w(q)}{\partial \theta_{3}}
\end{array}\right\}
\end{aligned}
$$

Here the aim of redundancy resolution is to maximize objective function i.e. manipulability. So inverse kinematics equation for redundancy resolution in the sense of manipulability maximization becomes 


$$
\Delta \theta=\mathrm{J}^{+} \overrightarrow{\mathrm{e}}+\left(\mathrm{I}-\mathrm{J}^{+} \mathrm{J}\right) \cdot\left\{\begin{array}{c}
\frac{\partial \mathrm{w}(\mathrm{q})}{\partial \theta_{1}} \\
\frac{\partial \mathrm{w}(\mathrm{q})}{\partial \theta_{2}} \\
\frac{\partial \mathrm{w}(\mathrm{q})}{\partial \theta_{3}}
\end{array}\right\}
$$

Manipulability is the function of three joint angles $\theta_{1}, \theta_{2}$ and $\theta_{3}$.

\section{Output and Observations}

A 3-R planar manipulator can reach at a point within the workspace with several possible configurations. For example, to reach the point $(2,5)$ end-effector may have various configurations as shown in Figure 12:

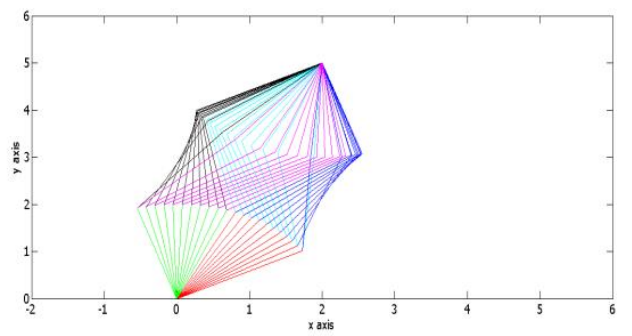

Fig. 12: Various Configurations of 3-R Planar Manipulator for a Target Point

Out of all possible configurations the best solution using damped least-square method is as shown in Figure 13:

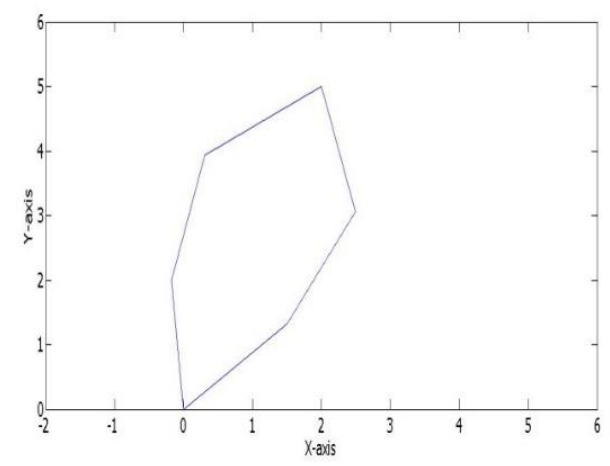

Fig. 13: Maximum Manipulability Configuration

Among all possible configurations for target point $(2,5)$, the above configuration of manipulator has maximum manipulability as shown in Figure 3.2. In the case of $3 \mathrm{R}$ redundant manipulator, there always exist two configurations for one manipulability value.

\section{Continuum Approach for slender structures}

The physical structure of such structure can be illustrated using the backbone curve having a form of:

$$
x(t)=\int_{0}^{t}[1+\varepsilon(\tau)] R(\tau) e_{1} d \tau
$$


Where $\mathrm{t} \varepsilon[0,1]$, and $\mathrm{e}_{1}=[1,0,0]^{\mathrm{T}}$ where $\mathrm{T}$ depicts transpose function. $\mathrm{R}(\tau) \mathrm{e}_{1}$ represents tangent vector, relative to $3 \times 3$ rotation matrix, $\mathrm{R}(\tau) \varepsilon \mathrm{BO}(3)$ for given values.

If a link interprets itself as a prismatic joint, then the degree though extent degree through it can be drawn represented as

$$
\frac{d x}{d t}=[1+\varepsilon(\mathrm{t})] R(t) \mathrm{e}_{1}
$$

Where magnitude of $\|\mathrm{dx} / \mathrm{dt}\|=1+\varepsilon(\mathrm{t})$, where, $\varepsilon(\mathrm{t}) \equiv 0$ and $\mathrm{t}$ is curve parameter. For redrawing the restricted curve using spline curve form using

$$
x(t)=\sum_{k=1}^{l} a_{i} \Phi_{i}
$$

Here shape function $\left(\Phi_{i}\right)$ through limit 1 to be equal to the degree of freedom for performing the task of certain operations. For restricting intrinsic parameters while observing realistic physical structure in slender shape mode to restrict in modal form, the planer equation represented as

$$
\mathrm{R}(\tau) \mathrm{e}_{1}=\left(\begin{array}{c}
\operatorname{cosine} \alpha(\tau) \\
\operatorname{sine} \alpha(\tau)
\end{array}\right)
$$

Here $\alpha$ is correlated with plane curvature under generalized formulation

$$
\alpha(\tau)=[\epsilon(\tau)+1] \lambda(\tau) d \tau
$$

Shows exemplary solution of the initial point (at $\epsilon(\tau)=0$ ) equations can be evaluated as,

$$
\varphi_{1}=2 \pi \cos 2 \pi \tau \text { and } \varphi_{2}=2 \pi \sin 2 \pi \tau
$$

Resulting in,

$$
\begin{gathered}
\Omega_{1}= \pm\left(\left[k_{0}^{-1}\left[x_{e}^{2}+y_{e}^{2}\right]^{\frac{1}{2}}\right]\right]^{2}-\left[k \tan 2\left(x_{e}+y_{e}\right)^{2}\right]^{1 / 2} \\
\Omega_{2}=k \tan 2\left(x_{e}+y_{e}\right)
\end{gathered}
$$

\section{Curve fitting:}

The curve fitting for the real manipulation devices moves with the information regarding orientation, path curves, and references at initial and final positions to arm respectively. So the required curves follow the movement as

$$
c(t)=(R(t) ; x, y(t)) \epsilon O(\varepsilon) * \mathbb{R}^{3}
$$

Where $\mathrm{O}(3)$ forms set of all group pair( Special Euclidian). The position and orientation are represented as

$$
c(t)^{T} R\left(t_{i+1}\right)=\left(R(t)^{T} R\left(t_{i+1}\right)\right)\left[R(t)^{T}\right]
$$

While interpreting such equations into $4 \times 4$ matrix homogeneous form, it may be drawn as

Such that,

$$
J(R, x)=\left(\begin{array}{cc}
R & x \\
O^{T} & 1
\end{array}\right)
$$

$$
\omega=\left(R \cdot R^{T}\right)
$$

and representing in matrx formulation where, $\Omega x=w X x$, explaining through example,

So,

$$
J(c)=\left(\begin{array}{cccc}
\cos \theta & -\sin \theta & 0 & 0 \\
\sin \theta & \cos \theta & 0 & 0 \\
0 & 0 & 1 & z \\
0 & 0 & 0 & 1
\end{array}\right)
$$




$$
\left(\begin{array}{l}
\omega \\
v
\end{array}\right)=\left(\begin{array}{cccc}
0 & -\theta & 0 & 0 \\
\theta & 0 & 0 & 0 \\
0 & 0 & 1 & z \\
0 & 0 & 0 & 1
\end{array}\right)=\begin{aligned}
& 0 \\
& 0 \\
& \theta \\
& 0 \\
& z
\end{aligned}
$$

\section{Langarian Method:}

For Langarian formulation motion equation can be formulated for $\mathrm{n}$ no. of $\mathrm{DoF}$ is,

$$
\frac{d}{d t}\left(\frac{\delta T}{\delta \Phi}\right)-\frac{\delta T}{\delta \Phi}+\frac{\delta V}{\delta \Phi}=\zeta
$$

Where $\mathrm{T}$ is kinetic and $\mathrm{V}$ is the potential energy of $\Phi \mathrm{n}^{\prime}$ dimensional arrays.

A take the case in the coordinate-free atmosphere, the snake-like structure formulated as,

$$
J=\int_{t 2}^{t 1} f\left(g: g^{-1} g: 1\right) d t
$$

Can also be represented through $4 \times 4$ matrix structure.

Such that, in $E_{i}^{\prime}$ representing directional derivative and $F_{i j}^{n}$ represents dimensional structure constants, fixed at any given $\Omega$ structure

Though, $\mathrm{n}=4$ in $\mathrm{O}(3)$ is

$$
\left[E_{i}, E_{j}\right]=\sum_{n=1}^{m} F_{i j}^{n} E_{m}
$$

$$
\begin{aligned}
E_{1} & =\left(\begin{array}{cccc}
1 & 0 & 0 & 0 \\
0 & 0 & 0 & -1 \\
0 & 0 & 1 & 0 \\
0 & 0 & 0 & 1
\end{array}\right) \\
E_{2} & =\left(\begin{array}{cccc}
1 & 0 & 0 & 0 \\
0 & 1 & 0 & 0 \\
-1 & 0 & 1 & 0 \\
0 & 0 & 0 & 1
\end{array}\right) \\
E_{3} & =\left(\begin{array}{cccc}
1 & 0 & 0 & 0 \\
0 & 1 & 0 & 0 \\
0 & 0 & 1 & 0 \\
0 & -1 & 0 & 0
\end{array}\right) \\
E_{4} & =\left(\begin{array}{llll}
0 & 0 & -1 & 0 \\
0 & 1 & 0 & 0 \\
0 & 0 & 1 & 0 \\
0 & 0 & 0 & 1
\end{array}\right)
\end{aligned}
$$

So the derivative $\mathrm{O}(3)$ and function $\mathrm{f}(\mathrm{r})=\mathrm{r}_{11}$, so the derivative computed as,

$$
\frac{d}{d t} e_{1}^{T} R\left(\mathrm{I}+t E_{1}\right) e_{1} \|_{t . . n}=e_{1}^{T} R E w=0
$$

In globally optimize the combination problem and rearranging the global optimality through $\theta(\mathrm{t})$ $=\theta^{b}(t)+\varepsilon(\tau)$ where $\varepsilon(0)=\varepsilon(1)$ in the main equation can be interpreted as 


$$
\frac{1}{2} \int_{0}^{1}\|b+\dot{\varepsilon}\|^{2} w \cdot d t=\frac{1}{2} a^{T} w a+a^{T} w \int_{0}^{1} \dot{\varepsilon} d t+\frac{1}{2} \int_{0}^{1}\|\dot{\varepsilon}\|^{2} w d t
$$

\section{Curve Formulation:}

Curve re-parameterization and optimal roll distribution arc length -wise parameterized using curve $\mathbf{x}(s)$ where $\mathrm{s}$ lies in boundaries $\mathrm{s} \in[0,1]$, and frames depicting Frenet-Serret apparatus. If Frenet frames denoted as $(F T(s), \mathbf{x}(s))$, then a novel set containing smooth evolving reference are $(P(t), x(t))=(F T t(s) R 1(\theta(t(s)))$ in the main equation can be interpreted as

$$
\left.F T=\frac{1}{2} \int_{0}^{1}\left\{\frac{1}{2} r^{2} s r\left(\dot{P} \dot{P}^{T}\right)+\dot{x} \cdot x\right\} d t=\frac{1}{2} \int_{0}^{1}\left\{r^{2} \kappa^{2}(s)+1\right\} \dot{s}+r^{3}(\tau(s) \dot{r}+\theta)^{2}\right) d t
$$

\section{Issues under the implementation of generated mechanisms:}

While generating possible and recognizable limits of the robotic arm still many limitations will be there to overcome in the next trends of researches are as follows

1. Permitting manipulator movement from one position to another may not be possible in realtime applications as obstacles and eminent parts play an important role in obstructions.

2. Programming v/s movement feedback will be observed on continuous fashion to avoid lagging in robotic manipulator placements.

3. Manipulability of arm to be examined and more simulation experiments to be carried out using feedback and tactile sensors for augmenting usability of a generated algorithm in industrial applications.

4. Graphical results in MATLAB represents changes in orientation and validation to such results needs verifications according to positioned specific motion taken for validations.

5. Standard algorithms can be possessed using these validated results on a wider fashion according to requirements using Robotic simulators.

6. Using continuum approach complex paths can be simplified, thus programming helps in attaining variable modes in lesser duration.

7. Matrix simulation of such achieved parameters came linked, thus output position can be calculated.

\section{Conclusions:}

While deriving such algorithms following conclusions can be made:

1. An idea to generalizing 3R movement of any arm shortest path selection can be achieved.

2. Examining various parameters here, for DLS simulations other dynamic simulations can be done using these algorithms changing their parameter variability.

3. Redundancy and manipulability can be attained in the precise way of consuming these algorithms.

4. Converting experimented and simulated results into programmable technique lead to the mature better simulator with path optimization and calculative structures.

5. Continuum approach makes the notation more complex as many indicative parameters are cross-linked, making the algorithm difficult to understand.

6. We can replace the simulated parameters with human-made and later examine the gathered results. 


\section{REFERENCES}

[1] S. Tejomurtula and S. J. I. S. Kak, "Inverse kinematics in robotics using neural networks," vol. 116, no. 2-4, pp. 147-164, 1999.

[2] R. Rao, A. Asaithambi, and S. J. J. o. M. D. Agrawal, "Inverse kinematic solution of robot manipulators using interval analysis," vol. 120, no. 1, pp. 147-150, 1998.

[3] R. J. Webster III, J. M. Romano, and N. J. J. I. T. o. R. Cowan, "Mechanics of precurved-tube continuum robots," vol. 25, no. 1, pp. 67-78, 2008.

[4] D. E. J. N. S. P. Whitney, "Resolved Motion Rate Control of Manipulators and Human Prostheses," vol. 215, p. $685,1970$.

[5] J. Baillieul, "Kinematic programming alternatives for redundant manipulators," in Proceedings. 1985 IEEE International Conference on Robotics and Automation, 1985, vol. 2, pp. 722-728: IEEE.

[6] C. A. Klein, C. Chu-Jenq, S. J. I. T. o. R. Ahmed, and Automation, "A new formulation of the extended Jacobian method and its use in mapping algorithmic singularities for kinematically redundant manipulators," vol. 11, no. 1, pp. 50-55, 1995.

[7] T. Yoshikawa, "Manipulability and redundancy control of robotic mechanisms," in Proceedings. 1985 IEEE International Conference on Robotics and Automation, 1985, vol. 2, pp. 1004-1009: IEEE.

[8] S. J. I. T. o. R. Chiaverini and Automation, "Singularity-robust task-priority redundancy resolution for realtime kinematic control of robot manipulators," vol. 13, no. 3, pp. 398-410, 1997.

[9] S. Chiaverini, B. Siciliano, and O. J. I. T. o. c. s. t. Egeland, "Review of the damped least-squares inverse kinematics with experiments on an industrial robot manipulator," vol. 2, no. 2, pp. 123-134, 1994.

[10] S. B. Slotine, "A general framework for managing multiple tasks in highly redundant robotic systems," in proceeding of 5th International Conference on Advanced Robotics, 1991, vol. 2, pp. 1211-1216.

[11] S. J. S.-L. L. Hirose and Manipulators, "Biologically inspired robots," 1993.

[12] S. Ma and N. J. A. R. Tadokoro, "Analysis of creeping locomotion of a snake-like robot on a slope," vol. 20, no. 1, pp. 15-23, 2006.

[13] S. J. A. Robotics, "Analysis of creeping locomotion of a snake-like robot," vol. 15, no. 2, pp. 205-224, 2001.

[14] A. Wolf et al., "A mobile hyper redundant mechanism for search and rescue tasks," in Proceedings 2003 IEEE/RSJ International Conference on Intelligent Robots and Systems (IROS 2003)(Cat. No. 03CH37453), 2003, vol. 3, pp. 2889-2895: IEEE.

[15] N. Simaan et al., "Design and integration of a telerobotic system for minimally invasive surgery of the throat," vol. 28, no. 9, pp. 1134-1153, 2009.

[16] N. Simaan, R. Taylor, and P. Flint, "A dexterous system for laryngeal surgery," in IEEE International Conference on Robotics and Automation, 2004. Proceedings. ICRA'04. 2004, 2004, vol. 1, pp. 351-357: IEEE.

[17] R. J. Murphy, M. S. Moses, M. D. Kutzer, G. S. Chirikjian, and M. Armand, "Constrained workspace generation for snake-like manipulators with applications to minimally invasive surgery," in 2013 IEEE International Conference on Robotics and Automation, 2013, pp. 5341-5347: IEEE.

[18] W. Park, J. S. Kim, Y. Zhou, N. J. Cowan, A. M. Okamura, and G. S. Chirikjian, "Diffusion-based motion planning for a nonholonomic flexible needle model," in Proceedings of the 2005 IEEE International Conference on Robotics and Automation, 2005, pp. 4600-4605: IEEE.

[19] S. Neppalli, M. A. Csencsits, B. A. Jones, and I. D. J. A. R. Walker, "Closed-form inverse kinematics for continuum manipulators," vol. 23, no. 15, pp. 2077-2091, 2009.

[20] G. S. J. A. R. Chirikjian, "Hyper-redundant manipulator dynamics: A continuum approximation," vol. 9, no. 3, pp. 217-243, 1994.

[21] P. E. Dupont, J. Lock, B. Itkowitz, and E. J. I. T. o. R. Butler, "Design and control of concentric-tube robots," vol. 26, no. 2, pp. 209-225, 2009.

[22] J. Li, Z. Teng, J. Xiao, A. Kapadia, A. Bartow, and I. Walker, "Autonomous continuum grasping," in 2013 IEEE/RSJ International Conference on Intelligent Robots and Systems, 2013, pp. 4569-4576: IEEE.

[23] G. Robinson and J. B. C. Davies, "Continuum robots-a state of the art," in Proceedings 1999 IEEE international conference on robotics and automation (Cat. No. 99CH36288C), 1999, vol. 4, pp. 2849-2854: IEEE. 
[24] G. S. Chirikjian and J. W. Burdick, "An obstacle avoidance algorithm for hyper-redundant manipulators," in Proceedings., IEEE International Conference on Robotics and Automation, 1990, pp. 625-631: IEEE.

[25] R. J. Webster III, J. S. Kim, N. J. Cowan, G. S. Chirikjian, and A. M. J. T. I. J. o. R. R. Okamura, "Nonholonomic modeling of needle steering," vol. 25, no. 5-6, pp. 509-525, 2006.

[26] I. D. Walker et al., "Continuum robot arms inspired by cephalopods," in Unmanned Ground Vehicle Technology VII, 2005, vol. 5804, pp. 303-314: International Society for Optics and Photonics.

[27] A. E. H. Love, A treatise on the mathematical theory of elasticity. Cambridge university press, 2013.

[28] H. J. P. o. t. R. S. o. E. S. A. M. Daniels, "XXI.-the statistical theory of stiff chains," vol. 63, no. 3, pp. 290311, 1952.

[29] J. Hermans and R. J. P. Ullman, "The statistics of stiff chains, with applications to light scattering," vol. 18, no. 11, pp. 951-971, 1952.

[30] O. Kratky and G. J. R. d. T. C. d. P. B. Porod, "Röntgenuntersuchung gelöster fadenmoleküle," vol. 68, no. 12, pp. 1106-1122, 1949.

[31] W. Gobush, H. Yamakawa, W. Stockmayer, and W. J. T. J. o. C. P. Magee, "Statistical mechanics of wormlike chains. I. Asymptotic behavior," vol. 57, no. 7, pp. 2839-2843, 1972.

[32] P. J. P. R. L. Nelson, "Sequence-disorder effects on DNA entropic elasticity," vol. 80, no. 26, p. 5810, 1998.

[33] J. D. Moroz and P. J. P. o. t. N. A. o. S. Nelson, "Torsional directed walks, entropic elasticity, and DNA twist stiffness," vol. 94, no. 26, pp. 14418-14422, 1997.

[34] R. Alterovitz, A. Lim, K. Goldberg, G. S. Chirikjian, and A. M. Okamura, "Steering flexible needles under Markov motion uncertainty," in 2005 IEEE/RSJ International Conference on Intelligent Robots and Systems, 2005, pp. 1570-1575: IEEE.

[35] J. F. Marko and E. D. J. M. Siggia, "Bending and twisting elasticity of DNA," vol. 27, no. 4, pp. 981-988, 1994.

[36] S. D. Levene and D. M. J. J. o. m. b. Crothers, "Ring closure probabilities for DNA fragments by Monte Carlo simulation," vol. 189, no. 1, pp. 61-72, 1986.

[37] N. G. Cheng et al., "Design and analysis of a robust, low-cost, highly articulated manipulator enabled by jamming of granular media," in 2012 IEEE International Conference on Robotics and Automation, 2012, pp. 4328-4333: IEEE.

[38] P. Dario et al., "A novel mechatronic tool for computer-assisted arthroscopy," vol. 4, no. 1, pp. 15-29, 2000.

[39] H.-S. Yoon, H.-J. Cha, J. Chung, and B.-J. Yi, "Compact design of a dual master-slave system for maxillary sinus surgery," in 2013 IEEE/RSJ International Conference on Intelligent Robots and Systems, 2013, pp. 5027-5032: IEEE.

[40] B. L. Conrad and M. R. Zinn, "Interleaved continuum-rigid manipulation approach: Development and functional evaluation of a clinical scale manipulator," in 2014 IEEE/RSJ International Conference on Intelligent Robots and Systems, 2014, pp. 4290-4296: IEEE.

[41] B. L. Conrad, J. Jung, R. S. Penning, and M. R. Zinn, "Interleaved continuum-rigid manipulation: An augmented approach for robotic minimally-invasive flexible catheter-based procedures," in 2013 IEEE International Conference on Robotics and Automation, 2013, pp. 718-724: IEEE.

[42] K. Xu, M. Fu, and J. Zhao, "An experimental kinestatic comparison between continuum manipulators with structural variations," in 2014 IEEE International Conference on Robotics and Automation (ICRA), 2014, pp. 3258-3264: IEEE.

[43] K. Xu, J. Zhao, and M. J. I. A. T. o. M. Fu, "Development of the SJTU unfoldable robotic system (SURS) for single port laparoscopy," vol. 20, no. 5, pp. 2133-2145, 2014.

[44] M. D. Kutzer, S. M. Segreti, C. Y. Brown, M. Armand, R. H. Taylor, and S. C. Mears, "Design of a new cabledriven manipulator with a large open lumen: Preliminary applications in the minimally-invasive removal of osteolysis," in 2011 IEEE International Conference on Robotics and Automation, 2011, pp. 2913-2920: IEEE.

[45] D. B. Camarillo, C. F. Milne, C. R. Carlson, M. R. Zinn, and J. K. J. I. T. o. R. Salisbury, "Mechanics modeling of tendon-driven continuum manipulators," vol. 24, no. 6, pp. 1262-1273, 2008.

[46] J. Till, C. E. Bryson, S. Chung, A. Orekhov, and D. C. Rucker, "Efficient computation of multiple coupled Cosserat rod models for real-time simulation and control of parallel continuum manipulators," in 2015 IEEE International Conference on Robotics and Automation (ICRA), 2015, pp. 5067-5074: IEEE. 
[47] C. E. Bryson and D. C. Rucker, "Toward parallel continuum manipulators," in 2014 IEEE International Conference on Robotics and Automation (ICRA), 2014, pp. 778-785: IEEE.

[48] Y.-J. Kim, S. Cheng, S. Kim, and K. lagnemma, "Design of a tubular snake-like manipulator with stiffening capability by layer jamming," in 2012 IEEE/RSJ International Conference on Intelligent Robots and Systems, 2012, pp. 4251-4256: IEEE.

[49] R. Kang, D. T. Branson, T. Zheng, E. Guglielmino, D. G. J. B. Caldwell, and biomimetics, "Design, modeling and control of a pneumatically actuated manipulator inspired by biological continuum structures," vol. 8, no. 3, p. 036008, 2013.

[50] J. Li and J. Xiao, "Progressive generation of force-closure grasps for an n-section continuum manipulator," in 2013 IEEE International Conference on Robotics and Automation, 2013, pp. 4016-4022: IEEE.

[51] Y.-J. Kim, S. Cheng, S. Kim, and K. J. I. t. o. r. lagnemma, "A stiffness-adjustable hyperredundant manipulator using a variable neutral-line mechanism for minimally invasive surgery," vol. 30, no. 2, pp. 382-395, 2013.

[52] M. S. Moses, M. D. Kutzer, H. Ma, and M. Armand, "A continuum manipulator made of interlocking fibers," in 2013 IEEE International Conference on Robotics and Automation, 2013, pp. 4008-4015: IEEE.

[53] A. Jiang, G. Xynogalas, P. Dasgupta, K. Althoefer, and T. Nanayakkara, "Design of a variable stiffness flexible manipulator with composite granular jamming and membrane coupling," in 2012 IEEE/RSJ International Conference on Intelligent Robots and Systems, 2012, pp. 2922-2927: IEEE.

[54] K. B. Reed et al., "Robot-assisted needle steering," vol. 18, no. 4, pp. 35-46, 2011.

[55] J. Xiao and R. Vatcha, "Real-time adaptive motion planning for a continuum manipulator," in 2010 IEEE/RSJ International Conference on Intelligent Robots and Systems, 2010, pp. 5919-5926: IEEE.

[56] B. A. Jones and I. D. J. I. T. o. R. Walker, "Kinematics for multisection continuum robots," vol. 22, no. 1, pp. 43-55, 2006.

[57] H. Mochiyama, "Hyper-flexible robotic manipulators," in IEEE International Symposium on MicroNanoMechatronics and Human Science, 2005, 2005, pp. 41-46: IEEE.

[58] R. D. Kamien, T. C. Lubensky, P. Nelson, and C. S. J. E. O'Hern, "Direct determination of DNA twist-stretch coupling," vol. 38, no. 3, p. 237, 1997. 